\title{
Cellular glucose transport and glucotransporter 4 expression as a therapeutic target: clinical and experimental studies
}

\author{
Anna Czech, Paweł Piątkiewicz and Jan Tatoń \\ Chair and Department of Internal Medicine, and Diabetology, Second Medical Faculty, Warsaw Medical University \\ (Brodnowski Hospital), Warsaw, Poland
}

Received: 2009.02.10, Accepted: 2009.04.29, Published online: 2009.11.03

(C) L. Hirszfeld Institute of Immunology and Experimental Therapy, Wrocław, Poland 2009

\begin{abstract}
Introduction: The normalization of cellular glucose assimilation is the basic aim of metabolic therapy in type 2 diabetes mellitus (T2DM). It requires parallel changes in the process of cellular glucose transport (CGT). Therefore the level of CGT could be regarded as a therapeutic target for oral hypoglycemic drugs in T2DM. To explore this hypothesis, CGT levels before and after sulfonylurea therapy were investigated. Peripheral blood lymphocytes were used as a cell model for testing CGT.

Materials and Methods: CGT was assessed by experimental in vitro tests allowing timed comparative observation of the transport process during the incubation of lymphocytes with $2-\left[{ }^{3} \mathrm{H}(\mathrm{G})\right]$ glucose under basal conditions and after the addition of sulfonylurea or sulfonylurea plus insulin. The incubation tests were performed at baseline in 28 persons with newly diagnosed, therapy-naive T2DM and in 20 control subjects. In the diabetic patients the tests for CGT were repeated after 3 months of sulfonylurea therapy. The level of glucotransporter 4 (GLUT4) expression was also assessed by flow cytometry before and after the therapy.

Results: Before treatment, CGT was significantly lower in the subjects with T2DM. The cells responded to the addition of sulfonylurea by a moderate increase in CGT. This response was augmented by the addition of insulin to sulfonylurea in the culture medium.

Conclusions: The three-month therapy with sulfonylurea resulted in a significant increase in CGT in all types of culture tests. This sulfonylurea-related improvement in CGT was associated with a near normalization of GLUT4 expression in the cells.

Key words: sulfonylurea, cellular glucose transport, lymphocytes, GLUT4.

Corresponding author: Paweł Piątkiewicz, Chair and Department of Internal Medicine, and Diabetology, Second Medical Faculty, Warsaw Medical University (Brodnowski Hospital), Kondratowicza 8, 03-242 Warsaw, Poland, e-mail: piatkiewicz@op.pl
\end{abstract}

\section{INTRODUCTION}

Glucose transport defects in type 2 diabetes mellitus (T2DM) lead to impairment of the rate of glucose utilization by peripheral tissues. The first step of this complex process is cellular glucose transport (CGT), the properties of which in peripheral blood lymphocytes of diabetic patients are not known and information on glucose transporters in these cells is scarce and inconsistent.

As the lipid bilayer membrane of the majority of cells in the human body is impermeable to monosaccharides, glucose needs a biological transport system consisting of specialized transport proteins, the glucotrans- porters (GLUTs) (Kahn 1996; Shepherd and Kahn 1999). These proteins provide the physiological balance between glucose supply in cells and its further metabolism (Czech and Corvera 1999; Shulman 2000). CGT is a multistage process. Its individual stages are coordinated by specialized regulatory systems. Under physiological conditions the sensitive and quantitative coordination between the activity of the molecular CGT system and intracellular glucose metabolism is related to glycemia. Insulin is among the most important regulators of this relationship (Matthaei et al. 2000). In diabetes mellitus, however, this physiological regulation becomes impaired. Glucose utilization by cells is diminished despite hyperglycemia. The primary reason 
behind this impairment is insulin deficiency or a decrease in the peripheral cells' sensitivity to insulin, which could involve also the CGT system; in this way, a special form of insulin resistance may arise. The diagnosis and correction of such pathophysiological disturbances in CGT should therefore be an aim of therapy. It follows that CGT may be approached as an important target for therapeutic drug activity. This refers especially to insulin therapy and also to use of the oral betacytotropic sulfonylureas. This hypothesis can be supported by many observations which suggest that the activity of oral betacytotropic drugs is pleiotropic and not limited solely to the stimulation of beta cells. They produce many other therapeutically positive effects, among them a decrease in insulin resistance (Almer 1984; Fava et al. 2002; Gram et al. 1988; Itoh et al. 2003; Jennings et al. 1992; O'Brien et al. 2000). This may also involve the activity of the CGT system.

Therefore the clinically important questions arise as to:

1) whether hypoglycemic betacytotropic sulfonylurea derivatives influence the CGT system in a clinically significant and therapeutically important way;

2) whether these drugs exert their influence solely by stimulating insulin secretion or do they diminish hyperglycemia also in another way, i.e. by directly influencing CGT? To answer these pathophisiologically and clinically important questions, special experiments were designed and performed.

The investigations included:

- a comparative assessment of CGT in healthy volunteers and in type 2 diabetes patients who had not been treated previously in any way;

- investigation of the influence of T2DM treatment with a sulfonylurea compound on CGT;

- study of changes in the expression of GLUT4, the glucotransporter playing the most important role in CGT, that could occur as an effect of treatment with sulfonylureas.

As the cell model for in vitro testing, peripheral blood lymphocytes of patients with diabetes mellitus type 2 and healthy subjects were used.

\section{Study aims}

The study aimed specifically at:

I. determining CGT activity in cells using the model of peripheral blood lymphocytes. These experiments were done using originally designed incubation tests assessing CGT in vitro:

1. in a control group of healthy volunteers:

- under basic conditions: lymphocyte incubation without additives

- under stimulated conditions:

a) lymphocyte incubation with a sulfonylurea compound; for this purpose, gliclazide was used

b) lymphocyte incubation with addition of sulfonylurea and insulin

2. In a study group of patients with newly diagnosed T2DM not previously treated pharmacologically and with the same set of cell incubation tests as for the healthy volunteers:

a) before and

b) after 12 weeks of sulfonylurea therapy.

II. In addition, a the comparative flow-cytometric assessment of GLUT4 expression in the model cells, i.e. the lymphocytes of healthy subjects and T2DM patients as described above before and after 12 weeks of sulfonylurea therapy.

It was expected that the comparison of the CGT measurements according to this plan would allow assessing whether CGT is altered in T2DM under the influence of therapy with betacytotropic sulfonylureas.

\section{MATERIALS AND METHODS}

\section{Diabetes mellitus type 2 and control groups}

A group of 28 patients with diabetes mellitus type 2 who were not previously treated pharmacologically was qualified for the study. The clinical diagnosis of type 2 diabetes was made according to World Health Organization (WHO) criteria (Pulido et al. 1996). Qualification of the study population was accepted by the University Bioethics Council. The subjects chosen had no pathological disorders other than diabetes mellitus type 2 . The control group consisted of 20 healthy volunteers with no family history of diabetes. Qualification was based on a complete medical examination according to the full protocol of the Warsaw Medical University clinical standard.

An 8-point daily glucose profile was taken and $\mathrm{HbA}_{1 \mathrm{c}}$ determinations were performed in the T2DM patient group. In the control group an oral glucose tolerance test $(75.0 \mathrm{~g})$ was performed according to WHO. The results of the initial clinical examination of both groups are presented in Table 1.

\section{CGT assessment in peripheral blood lymphocytes in vitro}

Lymphocyte suspension samples were taken from the 20 healthy control subjects and the 28 T2DM patients, previously untreated for hyperglycemia, before and after 3 month of sulfonylurea therapy and tested according to the study plan.

The influence of sulfonylurea treatment on CGT was studied in three independent series of lymphocyte incubation tests, i.e. with neither sulfonylurea nor insulin added, with sulfonylurea added, and with both sulfonylurea and insulin added. The CGT level was measured at 15, 30, and $60 \mathrm{~min}$ of incubation. In all cases and samples, the viability of lymphocytes was tested with the trypan blue method.

\section{GLUT4 expression}

GLUT4 expression was measured in the lymphocytes of the same subjects in whom CGT was determined, 
Table 1. Comparative clinical characteristic of the two study population groups: diabetes mellitus type 2 and control group

\begin{tabular}{|c|c|c|c|}
\hline Parameter & $\begin{array}{l}\text { Diabetes mellitus type } 2 \text { group } \\
\text { (before treatment) } \\
n=28\end{array}$ & $\begin{array}{l}\text { Control group } \\
n=20\end{array}$ & $\mathrm{p}$ \\
\hline & mean $(\mathrm{SD})$ & mean (SD) & \\
\hline Age, years & $58.0(70)$ & $48.6(11.4)$ & 0.003 \\
\hline Sex, M/F & $20 / 8$ & $8 / 12$ & \\
\hline $\mathrm{BMI}, \mathrm{kg} / \mathrm{m}^{2}$ & $28.49(2.21)$ & $27.68(2.44)$ & 0.235 \\
\hline \multicolumn{4}{|l|}{ Blood pressure, $\mathrm{mmHg}$ : } \\
\hline systolic & $135(14.8)$ & $127.3(13.3)$ & 0.060 \\
\hline diastolic & $79(8.0)$ & $77.0(9.2)$ & 0.365 \\
\hline Resting heart rate (min) & $80.0(7.4)$ & $80.4(8.7)$ & 0.865 \\
\hline $\mathrm{FPG}, \mathrm{mmol} / \mathrm{l}$ & $8.05(1.03)$ & $5.13(0.66)$ & $<0.001$ \\
\hline FSI, $\mu \mathrm{U} / \mathrm{ml}$ & $12.15(7.88)$ & $7.92(3.76)$ & 0.019 \\
\hline HOMA-IR & $4.368(2.806)$ & $1.844(1.008)$ & $<0.001$ \\
\hline $\mathrm{HbA}_{1 \mathrm{c}}(\%)$ & $6.83(0.86)$ & & - \\
\hline Creatinine, serum, mg/dl & $1.020(0.310)$ & $0.944(0.177)$ & 0.291 \\
\hline AspAT, IU/dl & $25.5(8.1)$ & $20.6(5.9)$ & 0.024 \\
\hline $\mathrm{AlAT}, \mathrm{IU} / \mathrm{dl}$ & $26.9(13.6)$ & $24.0(10.4)$ & 0.426 \\
\hline
\end{tabular}

FPG - fasting plasma glucose, FSI - fasting serum insulin, HOMA-IR - homeostatic model assessment-insulin resistance, AspAT - aspartate transaminase, serum, AlAT - alanine transaminase, serum.

which included 10 healthy control subjects, and 10 T2DM patients before and after sulfonylurea therapy.

\section{Measurement of deoxy-D-glucose uptake by lymphocytes}

The incubation tests were performed according to previously described methods (Curto et al. 1997; Kaliman et al. 1995; Szablewski et al. 2000) adapted to our laboratory. To $290 \mu \mathrm{l}$ of a suspension containing 300,000 lymphocytes, $1.5 \mu \mathrm{l}$ of deoxy-D-glucose-2-[ $\left.{ }^{3} \mathrm{H}(\mathrm{G})\right]$ (185-370 GBq (7,70000 Cii/mmol) (NEN Life Science Products, Inc.) and $7.5 \mu \mathrm{l}$ of phosphate-buffered saline solution (PBS) were added. Deoxy-D-glucose-2-[ $\left.{ }^{3} \mathrm{H}(\mathrm{G})\right]$ uptake was measured after 15, 30, and 60 min of incubation, after which the transport was stopped by addition of 2 vol of ice-cold, $50 \mathrm{mM}$ glucose in PBS. Then the lymphocytes were washed three times in the same solution and the cells were lysed with $0.1 \mathrm{mM} \mathrm{NaOH} / 0.1 \%$ SDS (sodium dodecylsulfate). The following day, after cell lyses (within $24 \mathrm{~h}$ ), radioactivity was determined by a scintillation counter (Wallac 1450 MicroBeta Trilux). Each procedure was performed in triplicate.

\section{GLUT4 expression measurements with flow cytometry}

Mononuclear cells were isolated from the blood samples on Gradisol L (fluid density: of $1.077 \mathrm{~g} / \mathrm{l}$; "AQUA-MEDICA") and washed twice in $0.9 \% \mathrm{NaCl}$. To mark the population of cells that presents GLUT4 protein expression, anti-GLUT4 monoclonal antibody was used together with one-color indirect immunofluorescence.

For every single staining process, a sample of $10^{6}$ mononuclear cells was taken. The cells were incubated for $30 \mathrm{~min}$ with $2 \mu \mathrm{l}$ of anti-GLUT4 antibody in an ice bath of $4^{\circ} \mathrm{C}$ and then washed with PBS plus $0.01 \%$ $\mathrm{NaN}_{3}$. The cells were then incubated for 20 min (ice bath, $4^{\circ} \mathrm{C}$ ) with $10 \mu \mathrm{l}$ of secondary nonspecific antibody binding to the immunoglobulin fragment $\mathrm{F}(\mathrm{ab})_{2}$ and conjugated with the fluorochrome fluorescein isothiocyanate (FITC). Later the MNCs were washed again with PBS plus $0.01 \% \mathrm{NaN}_{3}$ and suspended in $500 \mu \mathrm{l}$ of FACS Flow (Becton-Dickinson, USA).

For data acquisition and analysis, a FACSCalibur flow cytometer (Becton-Dickinson, USA) with CellQuest software (Becton-Dickinson, USA) was used. The results are given as the percentage of cells presenting GLUT4 expression (Piątkiewicz et al. 2007).

\section{Viability test}

The trypan blue test showed that the experimental methods used did not influence lymphocyte viability. Individual dead cells were observed in the samples both before and after the experiments. The amount of dead cells was the same in samples from the control subjects and from the T2DM patients. The type of hypoglycemic treatment administered also did not influence lymphocyte viability.

\section{Statistical analysis}

Comparison of the clinical and demographic parameters of the groups was performed using the MannWhitney or chi-squared tests as appropriate. Changes in the values of diabetes control parameters $\left(\mathrm{HbA}_{1 \mathrm{c}}, \mathrm{FPG}\right.$, FSI, HOMA-IR) between the week 0 (W0) and week 12 (W12) visits were investigated with the Wilcoxon test. Variations in glucose uptake in the peripheral blood 
lymphocytes were analyzed with the Mann-Whitney test (Armitage 1971).

\section{RESULTS}

CGT assessment in peripheral blood lymphocytes, control group and diabetes mellitus group before therapy with sulfonylurea $(\mathrm{WO})$

The incubation tests with the lymphocytes of the control subjects showed an active CGT. Its intensity clearly increased with the duration of incubation. The addition of sulfonylurea to the culture enhanced CGT by a statistically significant amount for the all investigated incubation times. The addition of sulfonylurea and insulin together intensified CGT at 15 and $30 \mathrm{~min}$. This did not occur at the incubation time of $60 \mathrm{~min}$ (Table 2).

The incubation tests with the lymphocytes of the diabetic patients showed that when sulfonylurea was not added to the incubation environment, the CGT values were lower than those in the healthy controls. The influences of sulfonylurea and sulfonylurea plus insulin added to the culture were distinctly weaker than in the healthy controls (Table 2).

The influence of sulfonylurea on CTG in peripheral blood lymphocytes after sulfonylurea therapy (W12)

Diabetes mellitus metabolic compensation parameters measured before the start of treatment and after 12 weeks of treatment with sulfonylureas significantly improved. Fasting plasma glucose concentration decreased significantly (on average by $1.77 \mathrm{mmol} / \mathrm{l}, \mathrm{p}<0.001$ ). This was associated with a decrease in $\mathrm{HbA}_{1 \mathrm{c}}$ level (on average by $0.69 \%, \mathrm{p}<0.001)$. Fasting plasma insulin concentration increased at the same time, on average by $2.65 \mu \mathrm{U} / \mathrm{ml}$ $(\mathrm{p}<0.022)$. The HOMA-IR index did not change.

CGT intensity was significantly and consistently higher 12 weeks after treatment with sulfonylurea than before, as shown in all the incubation tests. Increased glucose uptake was clearly observed. The CGT level after therapy was similar to that measured in the control group (Table 2).

\section{GLUT4 expression}

The results of GLUT4 expression in the peripheral blood lymphocytes of the control subjects and T2DM patients before and after 12 weeks of sulfonylurea treatment are presented in Table 3. As the results show, GLUT4 expression was observed in $0.6 \%$ of the lymphocytes of the control subjects. In T2DM patients this value was much higher $(15.5 \%)$ before treatment. Twelve weeks after the begin of sulfonylurea treatment, the level of GLUT4 expression diminished to a mean value of $2.7 \%$.

\section{DISCUSSION}

So far, the clinical study of CGT concerns only selected areas. It has, however, provided valuable infor-

Table 2. CGT intensity measured in incubation tests in vitro for peripheral blood lymphocytes taken from 20 control subjects and from type 2 diabetic patients (T2DP) at baseline and after the completion of 12 weeks gliclazide treatment, expressed in $\mathrm{pg} / 300,000$ lymphocytes

\begin{tabular}{|c|c|c|c|c|c|c|c|c|}
\hline \multirow[t]{2}{*}{ Sample } & \multirow[t]{2}{*}{ Model } & \multirow{2}{*}{$\begin{array}{l}\text { Incubation } \\
\text { time (min) }\end{array}$} & \multicolumn{6}{|c|}{ CGT intensity (pg/300,000 lymphocytes) } \\
\hline & & & mean & SD & mean & SD & mean & SD \\
\hline 1 & $\begin{array}{l}\text { Lymphocytes, } \\
\text { no drugs added }\end{array}$ & $\begin{array}{l}15^{\prime} \\
30^{\prime} \\
60^{\circ}\end{array}$ & $\begin{array}{l}123.1 \\
228.7 \\
442.8\end{array}$ & $\begin{array}{c}25.7 \\
58.8 \\
137.8\end{array}$ & $\begin{array}{l}106.4 \\
179.4 \\
263.1\end{array}$ & $\begin{array}{l}25.3 \\
32.3 \\
47.8\end{array}$ & $\begin{array}{l}162.4 \\
308.1 \\
543.6\end{array}$ & $\begin{array}{l}34.6 \\
57.5 \\
62.1\end{array}$ \\
\hline 2 & $\begin{array}{l}\text { Lymphocytes, } \\
\text { gliclazide added }\end{array}$ & $\begin{array}{l}15^{\prime} \\
30^{\prime} \\
60^{\circ}\end{array}$ & $\begin{array}{l}203.6 \\
417.1 \\
664.1\end{array}$ & $\begin{array}{c}49.1 \\
107.1 \\
186.8\end{array}$ & $\begin{array}{l}113.5 \\
199.0 \\
316.2\end{array}$ & $\begin{array}{l}25.8 \\
36.3 \\
57.8\end{array}$ & $\begin{array}{l}210.3 \\
412.0 \\
727.2\end{array}$ & $\begin{array}{c}36.7 \\
97.3 \\
147.5\end{array}$ \\
\hline 3 & $\begin{array}{l}\text { Lymphocytes, gliclazide } \\
\text { and insulin added }\end{array}$ & $\begin{array}{l}15 \\
30^{`} \\
60\end{array}$ & $\begin{array}{l}308.8 \\
647.7 \\
666.7\end{array}$ & $\begin{array}{c}87.2 \\
181.5 \\
186.4\end{array}$ & $\begin{array}{l}186.4 \\
293.3 \\
306.0\end{array}$ & $\begin{array}{l}28.4 \\
56.7 \\
57.1\end{array}$ & $\begin{array}{l}349.3 \\
689.6 \\
709.7\end{array}$ & $\begin{array}{c}54.5 \\
145.2 \\
150.0\end{array}$ \\
\hline & & \multicolumn{2}{|c|}{$\begin{array}{l}\text { Control subjects } \\
\mathrm{p}\end{array}$} & \multicolumn{2}{|c|}{$\begin{array}{c}\text { T2DP at baseline } \\
\mathrm{p}\end{array}$} & \multicolumn{3}{|c|}{$\begin{array}{c}\text { T2DP after } 12 \text { weeks } \\
\text { of gliclazide treatment } \\
\mathrm{p}\end{array}$} \\
\hline \multicolumn{2}{|c|}{ sample 2 vs. 1} & \multicolumn{2}{|c|}{$\begin{array}{l}15^{`}<0.001 \\
30^{`}<0.001 \\
60^{`}<0.001\end{array}$} & \multicolumn{2}{|c|}{$\begin{array}{l}15^{`}<0.118 \\
30^{`}<0.001 \\
60^{`}<0.001\end{array}$} & \multicolumn{3}{|c|}{$\begin{array}{l}15^{`}<0.001 \\
30^{`}<0.001 \\
60^{`}<0.001\end{array}$} \\
\hline \multicolumn{2}{|c|}{ sample 3 vs. 2} & \multicolumn{2}{|c|}{$\begin{array}{l}15^{`}<0.001 \\
30^{`}<0.001 \\
60^{`}<0.469\end{array}$} & \multicolumn{2}{|c|}{$\begin{array}{l}15^{`}<0.001 \\
30^{`}<0.001 \\
60^{`}<0.001\end{array}$} & \multicolumn{3}{|c|}{$\begin{aligned} & 15^{`}<0.001 \\
& 30^{`}<0.001 \\
& 60^{`}<0001\end{aligned}$} \\
\hline
\end{tabular}


Table 3. The percentage of peripheral blood lymphocytes presenting GLUT4 expression in control group and in type 2 diabetic patients before and after 12 weeks of gliclazide treatment

\begin{tabular}{|c|c|c|c|c|c|}
\hline \multicolumn{6}{|c|}{ Percentage of lymphocytes with GLUT4 expression } \\
\hline \multicolumn{2}{|c|}{ Control subjects } & \multicolumn{4}{|c|}{ T2DM patients } \\
\hline \multirow[t]{2}{*}{ mean } & \multirow[t]{2}{*}{ SD } & \multicolumn{2}{|c|}{ before gliclazide treatment (W0) } & \multicolumn{2}{|c|}{ after 12 weeks of gliclazide treatment (W12) } \\
\hline & & mean & SD & mean & SD \\
\hline 0.6 & 0.3 & 15.5 & 9.8 & 2.7 & 1.6 \\
\hline
\end{tabular}

$\mathrm{p}(\mathrm{W} 0$ vs $\mathrm{W} 12)=0.002$

mation regarding the activity and regulation of CGT and glucotransporter expression under various metabolic conditions, both physiological and pathological (Galuska et al. 1994; Piątkiewicz et al. 2007; Pulido et al. 1997; Szablewski et al. 2000; Tsiani et al. 1995). In the majority of studies, myocytes or adipocytes were used as the experimental model, while other cell models were practically ignored. The pharmacological aspects of CGT were also rarely addressed.

Peripheral blood cells, among them lymphocytes, constitute a model which has been shown to be very useful in pharmacological research (Chakrabarti et al. 1994; Daneman et al. 1992; Pulido et al. 1997; Pulido 2000; Vestergaard et al. 1995). They represent the overall CGT process. The investigation of glucose transport in peripheral blood cells is also interesting because it involves issues not yet fully ascertained and which may be clinically very significant due to lymphocytes' role and function (Estrada et al. 1994).

It is widely assumed that the increased susceptibility to infections observed in diabetes mellitus may be related to lymphocytic function abnormalities resulting from their pathological decrease of glucose metabolism (Piątkiewicz et al. 2007). This in turn may be due to impaired CGT (Estrada et al. 1994; Korgun et al. 2002) and abnormalities in the expression of glucotransporters.

Several studies have so far revealed that GLUTl, GLUT3, GLUT4, and GLUT9 are present in granulocytes and monocytes (Chakrabarti et al. 1994; Daneman et al. 1992; Dimitriadis et al. 2005; Joshi et al. 1999). GLUT1, GLUT3, and GLUT 9 expression has been shown in lymphocytes (Dimitriadis et al. 2005; Joshi et al. 1999; Otton et al. 2002). Surprisingly, in study results available to date, no GLUT4 expression in lymphocytes of healthy people has been observed (Longo and Elsas 1998; Phay et al. 2000). The expression of GLUT1 gene in lymphocytes from rat spleen increased under the influence of physical exercise (Phay et al. 2000). On the other hand, it has been shown that sustained hypoglycemia affects GLUT gene expression in human blood leukocytes, especially in granulocytes and monocytes. In granulocytes, the level of GLUT4 increases in this situation by $73 \%$ compared with control samples, whereas a simultaneous reduction in GLUT1 and GLUT3 is observed. No such effect has been observed in the lymphocyte population (Chakrabarti et al. 1994).
There are several examples of pharmacological research into CGT in experimental animals. Tsiani et al. investigated the influence of sulfonylurea on CGT intensity and on glucose transporter distribution in rat muscle tissue cells in vitro. They demonstrated that sulfonylurea causes a significant increase in CGT, independently of insulin. This correlated with an increase in GLUT1 expression in the plasma membrane of the tested cells (Piątkiewicz et al. 2007).

Interesting information concerning the influence of sulfonylurea on CGT in rat muscles was also presented by Pulido et al. (Galuska et al. 1994; Tsiani et al. 1995). Sulfonylurea increased CGT and enhanced GLUT4 translocation to the cell membrane. This direct effect was stimulated by insulin. A sulfonylurea compound administered to rats with streptozocine-induced diabetes mellitus normalized both GLUT4 expression and CGT in skeletal muscle tissue (Galuska et al. 1994).

The presented results of the CGT clinical therapeutic investigation with a lymphocyte model using incubation tests demonstrated abnormalities in CGT function in both untreated and sulfonylurea-treated type 2 diabetic patients. The experiments revealed that sulfonylurea therapy significantly affects CGT in a clinically significant way.

Comparison of the CGT values measured in the incubation tests at time periods of 15,30 , and $60 \mathrm{~min}$ in healthy subjects and T2DM patients both untreated and subjected to 12 weeks of sulfonylurea treatment provides important information on this issue. It was determined that in untreated T2DM patients the CGT values were always significantly lower than those of the healthy controls. The differences were statistically significant. In untreated T2DM patients, two phenomena coexisted: hyperglycemia and a significant decrease in CGT. This comparison validates the hypothesis that the CGT increase under the influence of sulfonylurea therapy may contribute to the decrease in hyperglycemia level in T2DM. This was confirmed by the observation of the effect of sulfonylurea therapy on CGT in the same patients before and after therapy. After 12 weeks of treatment with sulfonylurea, the previously decreased CGT level increased to a similar efficacy as that observed in the healthy controls. This therapeutic effect correlated with significant decreases in fasting glucose concentration and $\mathrm{HbA}_{1 \mathrm{c}}$ percentage. It may then be implied that sulfonylurea treatment increases CGT, simultaneously influencing these T2DM control 
parameters. It therefore constitutes a measure and a target of therapy.

Such a notion may be further substantiated by the observed changes in GLUT4 expression in lymphocytes induced at the same time by sulfonylurea treatment. They accompanied the CGT increase.

In the T2DM patients, two effects were noted simultaneously: increased CGT and decreased GLUT4 expression, both close to the values observed in healthy people. The decrease in GLUT4 expression was from a mean value of $15.5 \%$ to a mean value of $2.7 \%$ of cells $(p<0.001)$ as measured with flow cytometry. This is also definitely a positive therapeutic effect. Our observations suggest that sulfonylurea therapy also works at the level of peripheral cells, directly effecting the CGT system.

To summarize, the use of sulfonylurea drugs in T2DM treatment simultaneously exerted a positive pleiotropic therapeutic influence on both CGT intensity and GLUT4 expression.

In conclusion it could be stated, that: 1) the incubation tests showed that lymphocytes constitute a useful model for CGT investigations. In healthy subjects, the increase in CGT intensity is proportional to incubation time. CGT values in lymphocytes from healthy controls and tested in vitro also increase under the influence of sulfonylurea as well as under the influence of sulfonylurea and insulin together; 2) in pharmacologically untreated T2DM, CGT is diminished compared with healthy controls. This difference is statistically significant. The reaction of CGT to the addition of sulfonylurea and sulfonylurea together with insulin is also significantly reduced; 3) prolonged, 12-week sulfonylurea treatment causes a statistically significant increase in CGT in the studied cells; 4) in type 2 diabetic patients treated with sulfonylurea, the increase in CGT reaches the level encountered in healthy controls. It coexists with changes in GLUT4 expression. Under the influence of sulfonylurea therapy, the elevated GLUT4 expression before treatment decreases to the level observed in healthy controls.

\section{REFERENCES}

Almer LO (1984) Effects of chlorpropamide and gliclazide on plasminogen activator activity in vascular walls in patients with maturity onset diabetes. Thromb Res 35:19-25

Armitage P (1971) Statistical methods in medical research, 3rd ed. Blackwell Scientific Publication, Oxford

Chakrabarti R, Jung CY, Lee TP et al (1994) Changes in glucose transport and transporter isoforms during the activation of human peripheral blood lymphocytes by phytohemagglutinin. J Immunol 152:2660-2668

Curto M, Piccinini M, Rabbone I et al (1997) G proteins and regulation of pyruvate dehydrogenase activity by insulin in human circulating lymphocytes. Int $\mathrm{J}$ Biochem Cell Biol 29:1207-1217

Czech MP, Corvera S (1999) Signaling mechanisms that regulate glucose transport. J Biol Chem 274:1865-1868
Daneman D, Zinman B, Elliot ME et al (1992) Insulin-stimulated transport in circulating mononuclear cells from nondiabetic and IDDM subjects. Diabetes 41:227-234

Dimitriadis G, Maratou E, Boutati E et al (2005) Evaluation of glucose transport and its regulation by insulin in human monocytes using flow cytometry. Cytometry A 64:27-33

Estrada DE, Elliot E, Zinman B et al (1994) Regulation of glucose transport and expression of GLUT 3 transporters in human mononuclear cells; studies in cells from insulin-dependent diabetic and nondiabetic individuals. Metabolism 43:591-598

Fava D, Cassone-Faldetta M, Laurenti O et al (2002) Gliclazide improves anti-oxidant status and nitric oxide-mediated vasodilatation in type 2 diabetes. Diabet Med 19:752-757

Galuska D, Nolte LA, Zierath JR et al (1994) Effects of metformin on insulin-stimulated glucose transport in isolated skeletal muscle obtainer from patients with NIDDM. Diabetologia 37:826-832

Gram J, Jespersen J, Kold A (1988) Effects of an oral antidiabetic drug on the fibrinolytic system of blood in insulin-treated patients. Metabolism 37:937-943

Itoh M, Omi H, Okouchi M et al (2003) The mechanism of inhibitory actions of gliclazide on neutrophils-endothelial cells adhesion and surface expression of endothelial adhesion molecules mediated by a high glucose concentration. J Diabetes Complications 17:22-26

Jennings PE, Scott NA, Santabadi AR et al (1992) Effects of gliclazide on platelet reactivity and free radicals in type II diabetic patients: clinical assessments. Metabolism 41:36-39

Joshi N, Caputo GM, Weiteleamp MR et al (1999) Infection in patients with diabetes mellitus. N Engl J Med 341:1906-1912

Kahn BB (1996) Lilly lecture 1995. Glucose transport: pivotal step in insulin action. Diabetes 45:1644-1654

Kaliman P, Vinals F, Testar X et al (1995) Disruption of GLUT1 glucose carrier trafficking in L6E9 and So18 myoblast by the phosphatidylinositol 3-kinase inhibitor wortmannin. Biochem J 312:471-477

Korgun ET, Demir R, Sedlmayr P et al (2002) Sustained hypoglycemia affects glucose transporter expression of human blood leukocytes. Blood Cells Mol Dis 28:152-159

Longo M, Elsas LJ (1998) Human glucose transporters Adv Pediatr 45:293-313

Matthaei S, Stumvoll M, Kellerer M et al (2000) Pathophysiology and pharmacological treatment of insulin resistance. Endocr Rev 21:585-618

O'Brien RC, Luo M, Balazs N et al (2000) In vitro and in vivo antioxidant properties of gliclazide. J Diabetes Complications 14:201-206

Otton R, Mendonca JR, Curi R (2002) Diabetes causes marked changes in lymphocyte metabolism. $\mathrm{J}$ Endocrinol 174:55-61

Phay JE, Hussain HB, Moley JF (2000) Cloning and expression analysis of a novel member of the facilitative glucose transporters family, SLC2A9 (GLUT9). Genomics 66:217-220

Piątkiewicz P, Czech A, Tatoń J (2007) Glucose transport in 
human peripheral blood lymphocytes influenced by diabetes mellitus. Arch Immunol Ther Exp 55:119-126

Pulido N (2000) Gliclazide stimulates glucose transport in skeletal muscle through PKC activation. Diabetologia 43(suppl 1):706

Pulido N, Romero R., Suarez AI et al (1996) Sulfonylureas stimulate glucose uptake through GLUT4 transporter translocation in rat skeletal muscle. Biochem Biophys Res Commun 228:499-504

Pulido N, Suarez A, Casanova B et al (1997) Gliclazide treatment of streptozotocin diabetic rat restores GLUT4 protein content and basal glucose uptake in skeletal muscle. Metabolism 46(suppl 1):10-13

Shepherd PR, Kahn BB (1999) Glucose transporters and insulin action - implications for insulin resistance and diabetes mellitus. N Engl J Med 341:248-257
Shulman GI (2000) Cellular mechanism of insulin resistance. J Clin Invest 106:171-176

Szablewski L, Oleszczuk B, Malejczyk J et al (2000) Facilitative glucose transporters in rat chondrocycytes. Eur J Cell Biol 79(suppl 52):113

Tsiani E, Ramlal T, Leiter LA et al (1995) Stimulation of glucose uptake and increased plasma membrane content of glucose transporters in L6 skeletal muscle cells by the sulfonylureas gliclazide and glyburide. Endocrinology 136:2505-2512

Vestergaard H, Weinreb JE, Rosen AS et al (1995) Sulfonylurea therapy improves glucose disposal without changing skeletal muscle GLUT4 levels in noninsulin-dependent diabetes mellitus subjects: a longitudinal study. J Clin Endocrinol Metab 80:270-275 